\title{
Comparing the Ability and Accuracy of mSOFA, qSOFA, and qSOFA-65 in Predicting the Status of Nontraumatic Patients Referred to a Hospital Emergency Department: A Prospective Study
}

\author{
Abbasali Ebrahimian ${ }^{1}$, Seyyed-Mohammad-Taghi Shahcheragh², Ali Fakhr-Movahedi ${ }^{3}$
}

\begin{abstract}
Introduction: This study was proposed to compare the ability and accuracy of modified sequential organ failure assessment (mSOFA), quick SOFA (qSOFA), and qSOFA-65 in predicting the status of nontraumatic patients referred to hospital emergency departments (EDs).

Materials and methods: This study was a prospective design that performed on the 746 nontraumatic patients referred to the ED. Each patient data was collected using a demographic questionnaire, mSOFA, qSOFA, and qSOFA-65 scales. Related variables of each scale were recorded based on patients' medical records. Then, the outcome of each patient in the ED was followed up and recorded. The severity and specificity of each scale were estimated by the area under receiver operating characteristic (AUROC) curve at 99\% confidence interval (CI).

Results: The mean and standard deviation of scores were as follows: $\mathrm{mSOFA}=4.40 \pm 2.58, \mathrm{qSOFA}=0.50 \pm 0.70$, and $q \mathrm{SOFA}-65=0.92 \pm 0.96$. Patients requiring admission to the intensive care unit (ICU) were identified with $A U R O C$ curve as follows: $\mathrm{mSOFA}=0.882(99 \% \mathrm{Cl}=0.778-0.865)$; qSOFA $=0.717(99 \% \mathrm{Cl}=0.662-0.773)$; and qSOFA-65 $=0.771(99 \% \mathrm{Cl}=0.721-0.820)$, which showed that mSOFA has higher sensitivity and specificity than the other two scales in identifying patients requiring admission to the ICU.

Conclusion: All three scales were found to be reliable for identifying nontraumatic patients at risk of death and patients requiring admission to the ICU. However, since the time and data required to complete qSOFA and qSOFA-65 are much less than those of mSOFA, it is recommended that qSOFA and especially qSOFA- 65 be used in ED to identify critically ill nontraumatic patients.

Keywords: Emergency department, mSOFA, Nontraumatic patients, qSOFA, qSOFA-65, SOFA.

Indian Journal of Critical Care Medicine (2020): 10.5005/jp-journals-10071-23656
\end{abstract}

\section{INTRODUCTION}

Overcrowding of in the last two decades, ${ }^{1}$ studies have shown that emergency department (ED) overcrowding can increase the likelihood of morality within the next $1-3$ days, ${ }^{2}$ complications of diseases, ${ }^{3}$ delaying correct and effective treatment of patients, ${ }^{4}$ potential medical errors, ${ }^{5}$ hospital stay, ${ }^{6,7}$ health expenses, ${ }^{8}$ and a reduction in patient satisfaction. ${ }^{9}$ Over the last decade, many interventions have been conducted to reduce ED overcrowding, patients leaving the ward without being seen, and waiting time. Yet, ED overcrowding is still the main problem, and no reduction has been observed in the total time of patient stay in ED. ${ }^{10}$

One of the main reasons for the overcrowding of hospital EDs is referring to nontraumatic patients to the hospital ED, which accounts for over $80 \%$ of prehospital emergency referrals. ${ }^{11}$ Yet, not all nontraumatic patients presenting to or referred to hospital EDs require emergency services, and attending to patients according to the referral order is not an appropriate criterion for providing services in these wards. ${ }^{12}$ Hence, solutions have to think up to reduce mortality rates and other problems caused by overcrowding of hospital EDs.

An efficient method with fewer errors that can be useful for accurate identification of patients at risk is the scoring systems. One of the tools for identifying patients at risk in emergency and other hospital wards is a modified sequential organ failure assessment (mSOFA), which is the modified version of the SOFA that developed in 2007 by Grissom et al. ${ }^{13}$ In 2016, Seymour et al. introduced the

\begin{abstract}
${ }^{1}$ Nursing Care Research Center, Emergency Nursing Department, Semnan University of Medical Sciences, Semnan, Iran

${ }^{2}$ Student Research Committee, Faculty of Nursing, Semnan University of Medical Sciences, Semnan, Iran

${ }^{3}$ Nursing Care Research Center, Pediatric and Neonatal Nursing Department, Semnan University of Medical Sciences, Semnan, Iran

Corresponding Author: Ali Fakhr-Movahedi, Nursing Care Research Center, Pediatric and Neonatal Nursing Department, Semnan University of Medical Sciences, Semnan, Iran, Phone: +989122319538, e-mail: Alimovi49@semums.ac.ir
\end{abstract}

How to cite this article: Ebrahimian A, Shahcheragh SMT, FakhrMovahedi A. Comparing the Ability and Accuracy of mSOFA, qSOFA, and qSOFA-65 in Predicting the Status of Nontraumatic Patients Referred to a Hospital Emergency Department: A Prospective Study. Indian J Crit Care Med 2020;24(11):1045-1050.

Source of support: Nil

Conflict of interest: None

latest version of SOFA called quick SOFA (qSOFA), in which only respiratory rate, systolic blood pressure, and changes in the level of consciousness that used to identify critical patients suspected of infection in EDs. ${ }^{14}$ Over recent years, some studies have investigated the sensitivity and specificity of qSOFA in detecting the severity of infection in patients presenting to hospital EDs, and have reported conflicting results. Singer et al. showed that qSOFA is a suitable tool for identifying the possibility of death, the need for hospitalization 
in the intensive care unit (ICU), and the duration of hospital stay in patients suspected of infection in ED. ${ }^{15}$ Garbero et al. compared the sensitivity of SOFA and qSOFA in identifying patients suspected of infection and showed that qSOFA is not an appropriate tool for identifying patients with sepsis and its prognosis. ${ }^{16} \mathrm{~A}$ new version of qSOFA was introduced in 2020 by Jonghoo Lee and Jae-Uk Son, in which, age over 65 years was added to qSOFA as an effective variable in identifying patients suspected of infection in ED. They showed that qSOFA-65 is a better tool than qSOFA for identifying patients suspected of infection in ED. ${ }^{17}$ Some studies have used qSOFA for screening other patients. Jawa et al. studied qSOFA for determining the prognosis of trauma patients that showed the qSOFA could identify trauma patients at risk of death, the need for hospitalization in ICU, and the severity of injuries. ${ }^{18}$

It seems that the qSOFA and qSOFA-65 appear to be suitable for determining the status of nontraumatic patients dispatched to hospital EDs. However, researchers found no study on the use of these two scales to identify the likelihood of death, and the need for nontraumatic patients to stay in ICU or other wards. Thus, the present study was conducted to compare the ability of mSOFA, qSOFA, and qSOFA-65 in predicting the disposition type of nontraumatic patients referred to hospital EDs.

\section{Materials and Methods}

\section{Study Design, Setting, and Research Population}

This study was a prospective design to assess the ability and accuracy of mSOFA, qSOFA, and qSOFA- 65 in predicting the status of nontraumatic patients referred to hospital ED. The study was conducted over 6 months from late February to late August 2019. The study population included 923 nontraumatic patients who had been transferred by prehospital emergency to ED of kowsar hospital (a teaching-research hospital and a referral center for nontraumatic patients in Semnan province with 220 beds) in the city of Semnan. A total of 746 patients were ultimately selected purposively and nonrandomly according to inclusion and exclusion criteria. The study inclusion criteria were patients' informed consent, admission to ED, older than 18 years of age, and without trauma, or psychiatric disorders. The study exclusion criteria were the patient's unwillingness to continue cooperation with the study, patient transfer to another hospital, personal discharge against medical advice, and insufficient information for completing demographics, mSOFA, qSOFA, and qSOFA-65 questionnaires.

Patients were referred to the hospital ED and examined based on the chief complaint and received emergency treatment. Then, patients' disease was diagnosed based on the results of clinical and paraclinical tests. So, the chief complaint was considered as the initial patients' status in the admission time to the ED.

\section{Data Collection and Measurement}

At first, following their admission to hospital ED, patients were first visited by the emergency physicians or internists, and the physicians' instructions were carried out by emergency nurses. Then nurses performed the initial medical procedures.

Data were collected using a demographic questionnaire (age, gender, and marital status, education, duration of stay in ED, history of hospitalization, vital signs, and chief complaint) and mSOFA, qSOFA, and qSOFA-65 scales.

At first, the three scales were completed according to the values of the patient's records such as the first vital signs, the first oxygen-therapy request, the first dose of dopamine, dobutamine, or norepinephrine, the first scleral examination, and the first time creatinine level was measured in ED. Then, after the collection of the initial data, the patient outcome in the ED was followed up and recorded. The patients divided into four categories according to their status: (1) discharge from ED; (2) transfer to internal medicine wards; (3) transfer to ICU; and (4) death in ED. Also, the criterion for transferring patients from the ED to other wards was according to the opinion of emergency medicine and internal medicine specialists.

mSOFA assesses parameters relating to 5 vital organs, including the lungs, heart and vessels, liver, kidney, and central nervous system, and scores each organ from 0 to 4 points, with total score varying from 0 to 20 (Table 1). ${ }^{13}$ In qSOFA, SBP $\leq 100$ $\mathrm{mm} \mathrm{Hg}, \mathrm{RR} \geq 22$ breaths/minute, and GCS $\geq 14$ score 1 point, and the rest score 0 . Therefore, the final score of qSOFA has ranged between 0 and 3 points (14). In qSOFA-65, in addition to the existing values, 1 point is added to the mSOFA score for age over 65 years, making a total score of qSOFA- 65 from 0 to 4 points (16). All these three scales that used in this study have been reported with the confirmed validity and reliability in several studies to assess a variety of patients' critical conditions and to predict mortality $(13,14$, and 16$)$.

\section{Statistical Analysis}

Data were analyzed in SPSS-16 using descriptive (frequency, mean, and standard deviation) and analytical (Pearson and Spearman correlation coefficients) statistical tests and area under receiver operating characteristic (AUROC) curve at $99 \%$ confidence interval (CI). AUROC curve was used to determine the sensitivity and specificity of mSOFA, qSOFA, and qSOFA- 65 scales. Also, since the distribution of variables was abnormal based on the Shapiro-Wilk

Table 1: Modified sequential organ failure assessment (mSOFA) score

\begin{tabular}{|c|c|c|c|c|c|}
\hline Organ system & 0 & 1 & 2 & 3 & 4 \\
\hline Respiratory $\mathrm{SpO}_{2} / \mathrm{FiO}_{2}$ & $>400$ & $\leq 400$ & $\leq 315$ & $\leq 235$ & $\leq 150$ \\
\hline Liver & $\begin{array}{l}\text { No scleral icterus or } \\
\text { jaundice }\end{array}$ & & & $\begin{array}{l}\text { Scleral icterus or } \\
\text { jaundice }\end{array}$ & \\
\hline \multirow[t]{3}{*}{$\begin{array}{l}\text { Cardiovascular, } \\
\text { hypotension }\end{array}$} & No hypotension & $\mathrm{MAP}<70 \mathrm{~mm} \mathrm{Hg}$ & $\begin{array}{l}\text { Dopamine } \leq 5 \text { or } \\
\text { dobutamine any dose }\end{array}$ & Dopamine $>5$ & Dopamine $>15$ \\
\hline & & & & Epinephrine $\leq 0.1$ & Epinephrine $>0.1$ \\
\hline & & & & Norepinephrine $\leq 0.1$ & Norepinephrine $>0.1$ \\
\hline $\begin{array}{l}\text { CNS, Glasgow coma } \\
\text { scale }\end{array}$ & 15 & $13-14$ & $10-12$ & $6-9$ & $<6$ \\
\hline Renal, creatinine, mg/dL & $<1.2$ & $1.2-1.9$ & $2.0-3.4$ & $3.5-4.9$ & $>5.0$ \\
\hline
\end{tabular}


test, the distribution assumption was selected as nonparametric when analyzing the ROC curve.

\section{Ethical Considerations}

This study was approved by the research ethics committee of Semnan university of medical sciences, Semnan, Iran (No: IR.SEMUMS.REC.1397.280). The purpose of the study was explained to each patient. Also, informed consent was obtained from all patients. It was emphasized that the participation of each patient was voluntary, and they were free to withdraw from the study.

\section{Results}

Of the 746 patients, 71 patients (9.51\%) excluded for various reasons, including transfer to another hospital (9 patients), discharge from ED against medical advice (45 patients), age under 18 years (11 patients), and inadequate information needed to complete questionnaires (17 patients), so data relating to 664 patients were analyzed ultimately.

Of all participants, 360 patients (54.2\%) were males, and 304 (45.8\%) were females. Participants' mean age was $56.59 \pm 21.44$ years, and 535 patients (80.6\%) were married. The patients' mean to stay in the ED was $5.60 \pm 5.68$ hours. A total of 396 patients $(59.6 \%)$ had a history of hospitalization at least once, and 268 (40.4\%) had not to experience hospitalization. A total of hospitalized patients, 227 patients (34.2\%) were referred to the hospital for cardiovascular problems, 18 patients (2.7\%) died in ED, $110(16.6 \%)$ were transferred from ED to internal medicine ward, $110(16.6 \%)$ were transferred to ICU, and 426 (64.1\%) were discharged from ED (Table 2). Pearson correlation test showed a direct relationship between the length of stay in ED of nontraumatic patients and scores obtained from the three scales, that is, the higher the patients' scores, the longer their stay $(p<0.0001)$.

Mean and standard deviation of patients' vital signs and other medical measurements are as follows: systolic blood pressure 125.51 $\pm 29.44 \mathrm{~mm} \mathrm{Hg}$, diastolic blood pressure $76.98 \pm 16.75 \mathrm{~mm} \mathrm{Hg}$, mean atrial pressure (MAP) $93.34 \pm 19.63 \mathrm{~mm} \mathrm{Hg}$, heart rate $81.37 \pm 20.09$ bpm, respiratory rate $17.29 \pm 4.37 \mathrm{bpm}$, body temperature $36.83 \pm$ $0.71^{\circ} \mathrm{C}, \mathrm{O}_{2}$ saturation $94.58 \pm 7.57 \%$, GCS $13.94 \pm 2.67$, pain $1.56 \pm$ 3.04 , and blood glucose $137.85 \pm 75.54 \mathrm{mg} / \mathrm{dL}$ (Table 2).

The mean and standard deviation of scores was as follows: mSOFA $=4.40 \pm 2.58$, qSOFA $=0.50 \pm 0.70$, and qSOFA- $65=0.92 \pm$ 0.96 (Table 3). None of the patients with an mSOFA score less than 6 died. The odds of mortality and hospitalization in ICU increased with an increasing mSOFA score. All patients with qSOFA scores higher than 2, either died or were hospitalized. None of the patients with qSOFA- 65 score less than 3 died, and none with score greater than 3 were discharged. The cut-off point of the qSOFA scores for hospitalization in the ICU and death in the ED is 3 and 6, respectively.

Patients at risk of dying were identified with $A U R O C$ as follows: mSOFA $=0.923(99 \% \mathrm{Cl}=0.896-0.950)$; qSOFA $=0.925(99 \% \mathrm{Cl}=$ $0.885-0.965)$; and qSOFA-65 $=0.947$ ( $99 \% \mathrm{Cl}=0.922-0.973)$, which showed that mSOFA and qSOFA have equal sensitivities and specificities, and qSOFA-65 had more ability than the other two scales in detecting the likelihood of death (Fig. 1). Patients referred to the ICU were identified with AUROC as follows: $\mathrm{mSOFA}=0.882$ (99\% Cl = 0.778-0.865); qSOFA $=0.717$ (99\% Cl = 0.662-0.773); and qSOFA- $65=0.771(99 \% \mathrm{Cl}=0.721-0.820)$, which showed that mSOFA has higher sensitivity and specificity than the other two scales in identifying patients requiring admission to the ICU (Fig. 2). Patients referred to other wards were identified with $A U R O C$ as follows: $\mathrm{mSOFA}=0.569(99 \% \mathrm{Cl}=0.512-0.626)$; qSOFA $=0.491(99 \%$
Table 2: Background and physiological parameters of patients

\begin{tabular}{|c|c|c|}
\hline \multicolumn{2}{|c|}{ Demographic and physiological parameters } & $\begin{array}{l}\text { Frequency (\%) or } \\
\text { mean (SD) }\end{array}$ \\
\hline \multicolumn{2}{|c|}{ Age (year) } & $56.59 \pm 21.44$ \\
\hline \multicolumn{2}{|l|}{$\begin{array}{l}\text { Gender (male/fe- } \\
\text { male) }\end{array}$} & $360(54.2) / 304(45.8)$ \\
\hline \multicolumn{2}{|l|}{$\begin{array}{l}\text { Marriage status (mar- } \\
\text { ried/single) }\end{array}$} & $535(80.6) / 129(19.4)$ \\
\hline \multicolumn{2}{|l|}{$\begin{array}{l}\text { Hospitalization his- } \\
\text { tory (yes/no) }\end{array}$} & $\begin{array}{l}396(59.6 \%) / 268 \\
(40.4 \%)\end{array}$ \\
\hline \multirow{10}{*}{$\begin{array}{l}\text { Patients chief com- } \\
\text { plain, } n(\%)\end{array}$} & Neurological & $85(12.8)$ \\
\hline & Cardiovascular & $227(34.2)$ \\
\hline & Gastrointestinal & $52(7.8)$ \\
\hline & Respiratory & $56(8.4)$ \\
\hline & Renal & $2(0.3)$ \\
\hline & Endocrinological & $16(2.4)$ \\
\hline & Weakness & $75(11.3)$ \\
\hline & LOC & $56(8.4)$ \\
\hline & Poisoning & $64(9.6)$ \\
\hline & Other & $31(4.7)$ \\
\hline \multicolumn{2}{|l|}{$\begin{array}{l}\text { ED length of stay } \\
\text { (hours) }\end{array}$} & $5.60 \pm 5.68$ \\
\hline \multirow{4}{*}{$\begin{array}{l}\text { Patient's status in ED, } \\
n(\%)\end{array}$} & Discharge from ED & $426(64.1)$ \\
\hline & $\begin{array}{l}\text { Transport to general } \\
\text { ward }\end{array}$ & $110(16.6)$ \\
\hline & Transport to ICU & $110(16.6)$ \\
\hline & Death & $18(2.7)$ \\
\hline \multicolumn{2}{|l|}{$\mathrm{SBP}(\mathrm{mm} \mathrm{Hg})$} & $125.51 \pm 29.44$ \\
\hline \multicolumn{2}{|l|}{$\mathrm{DBP}(\mathrm{mm} \mathrm{Hg})$} & $76.98 \pm 16.75$ \\
\hline \multicolumn{2}{|l|}{ MAP (mm Hg) } & $93.34 \pm 19.63$ \\
\hline \multicolumn{2}{|l|}{$\mathrm{HR}(\mathrm{bpm})$} & $81.37 \pm 20.09$ \\
\hline \multicolumn{2}{|l|}{$\mathrm{RR}$} & $17.29 \pm 4.37$ \\
\hline \multicolumn{2}{|l|}{$\mathrm{T}$} & $36.83 \pm 0.71$ \\
\hline \multicolumn{2}{|l|}{$\mathrm{O}_{2}$ Saturation } & $94.58 \pm 7.57$ \\
\hline \multicolumn{2}{|l|}{ GCS } & $13.94 \pm 2.67$ \\
\hline \multicolumn{2}{|l|}{ Pain* based on VAS } & $1.56 \pm 3.04$ \\
\hline \multicolumn{2}{|l|}{ BS } & $137.85 \pm 75.54$ \\
\hline
\end{tabular}

*It refers to any pain that results from internal medicine conditions Data are shown as mean \pm standard deviation or $n$.

LOC, loss of consciousness; ED, emergency department; ICU, intensive care unit; SBP, systolic blood pressure; DBP, diastolic blood pressure; MAP, mean atrial pressure; $H R$, heart rate; bpm, beat per minute; $R R$, respiratory rate; $T$, temperature; GCS, Glasgow coma scale; VAS, visual analog scale; BS, blood sugar.

$\mathrm{Cl}=0.443-0.549)$; and qSOFA-65 $=0.510(99 \% \mathrm{Cl}=0.451-0.569)$, which showed that none of the three scales is superior to the other in sensitivity and specificity for identifying patients requiring transfer to other wards (Fig. 3).

\section{Discussion}

The present study was purposed to assess the ability and accuracy of mSOFA, qSOFA, and qSOFA-65 in identifying patients at risk of death, patients requiring admission to the ICU, and patients requiring transfer to other wards. 
Table 3: Distribution of mSOFA, qSOFA and qSOFA-65 scores in emergency patients

\begin{tabular}{|c|c|c|c|c|c|c|c|c|}
\hline \multirow[b]{2}{*}{ Scales } & \multirow[b]{2}{*}{ Scores } & \multirow[b]{2}{*}{$\begin{array}{l}\text { Frequency, } \\
n(\%)\end{array}$} & \multicolumn{4}{|c|}{ Patients status in emergency department } & \multirow[b]{2}{*}{ Mean $\pm S D$} & \multirow[b]{2}{*}{ Median (IQR } \\
\hline & & & $\begin{array}{l}\text { Discharge } \\
\text { from } E D, n(\%)\end{array}$ & $\begin{array}{l}\text { Transport to } \\
\text { general ward, } \\
n(\%)\end{array}$ & $\begin{array}{l}\text { Transport to } \\
I C U, n(\%)\end{array}$ & Death, $n(\%)$ & & \\
\hline \multirow[t]{18}{*}{ mSOFA } & 0 & $0(0)$ & $0(0)$ & $0(0)$ & $0(0)$ & $0(0)$ & $4.40 \pm 2.58$ & 3.70 \\
\hline & 1 & $0(0)$ & $0(0)$ & $0(0)$ & $0(0)$ & $0(0)$ & & \\
\hline & 2 & $139(20.8)$ & $119(85.6)$ & $18(12.9)$ & $2(1.5)$ & $0(0)$ & & \\
\hline & 3 & $156(23.4)$ & $129(82.7)$ & $17(10.9)$ & $10(6.4)$ & $0(0)$ & & \\
\hline & 4 & $170(25.6)$ & $119(70)$ & $31(18.2)$ & $20(11.8)$ & $0(0)$ & & \\
\hline & 5 & $57(8.6)$ & $31(54.4)$ & $16(28.1)$ & $10(17.5)$ & $0(0)$ & & \\
\hline & 6 & $38(5.7)$ & $15(39.5)$ & $12(31.6)$ & $10(26.3)$ & $1(2.6)$ & & \\
\hline & 7 & $23(3.5)$ & $3(13)$ & $5(21.7)$ & $9(39.1)$ & $6(26.1)$ & & \\
\hline & 8 & $22(3.3)$ & $6(27.3)$ & $4(18.2)$ & $11(50)$ & $1(4.5)$ & & \\
\hline & 9 & $13(2.0)$ & $0(0)$ & $4(30.8)$ & $6(46.2)$ & $3(23)$ & & \\
\hline & 10 & $20(3)$ & $4(20)$ & $0(0)$ & $14(70)$ & $2(10)$ & & \\
\hline & 11 & $5(0.8)$ & $0(0)$ & $1(20)$ & $2(40)$ & $2(40)$ & & \\
\hline & 12 & $8(1.2)$ & $0(0)$ & $1(12.5)$ & $6(75)$ & $1(12.5)$ & & \\
\hline & 13 & $6(0.9)$ & $0(0)$ & 1 (16.7) & $4(66.7)$ & 1 (16.7) & & \\
\hline & 14 & $5(0.8)$ & $0(0)$ & $0(0)$ & $4(80)$ & $120)$ & & \\
\hline & 15 & $1(0.2)$ & $0(0)$ & $0(0)$ & $1(100)$ & $0(0)$ & & \\
\hline & 16 & $1(0.2)$ & $0(0)$ & $0(0)$ & $1(100)$ & $0(0)$ & & \\
\hline & $>16$ & $0(0)$ & $0(0)$ & $0(0)$ & $0(0)$ & $0(0)$ & & \\
\hline \multirow[t]{4}{*}{ qSOFA } & 0 & 409 (60.6) & $305(75.5)$ & 67 (16.6) & $32(7.9)$ & $0(0)$ & $0.50 \pm 0.70$ & 0.43 \\
\hline & 1 & $192(28.4)$ & $106(56.4)$ & 35 (18.6) & $44(23.4)$ & $3(1.6)$ & & \\
\hline & 2 & $69(10.2)$ & $15(22.1)$ & $8(11.8)$ & $31(45.6)$ & $14(20.6)$ & & \\
\hline & 3 & $5(0.8)$ & $0(0)$ & $0(0)$ & $3(75)$ & $1(25)$ & & \\
\hline \multirow[t]{5}{*}{ qSOFA-65 } & 0 & $280(41.5)$ & $218(79.3)$ & $44(16)$ & $13(4.7)$ & $0(0)$ & $0.92 \pm 0.96$ & 0.77 \\
\hline & 1 & $230(34.1)$ & $160(70.8)$ & $36(15.9)$ & $30(13.3)$ & $0(0)$ & & \\
\hline & 2 & $107(15.8)$ & 42 (39.6) & $24(22.6)$ & 37 (34.9) & $3(2.8)$ & & \\
\hline & 3 & $56(8.3)$ & $6(10.9)$ & $6(10.9)$ & $28(50.9)$ & $15(27.3)$ & & \\
\hline & 4 & $2(0.3)$ & $0(0)$ & $0(0)$ & $2(100)$ & $0(0)$ & & \\
\hline
\end{tabular}

$\mathrm{SD}$, standard deviation; IQR, interquartile range

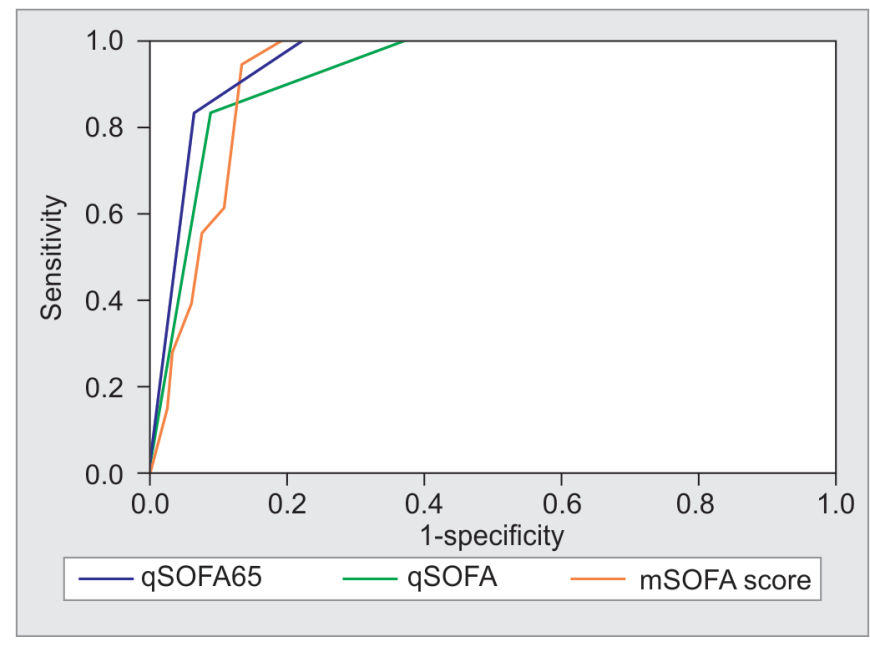

Fig. 1: mSOFA, qSOFA, and qSOFA-65 scales as detecting likelihood of death

The results showed that patients with mSOFA score $\geq 2$ needed ED admission for further assessments, those with mSOFA score $\geq 6$ needed provision of more care, and all those with mSOFA score

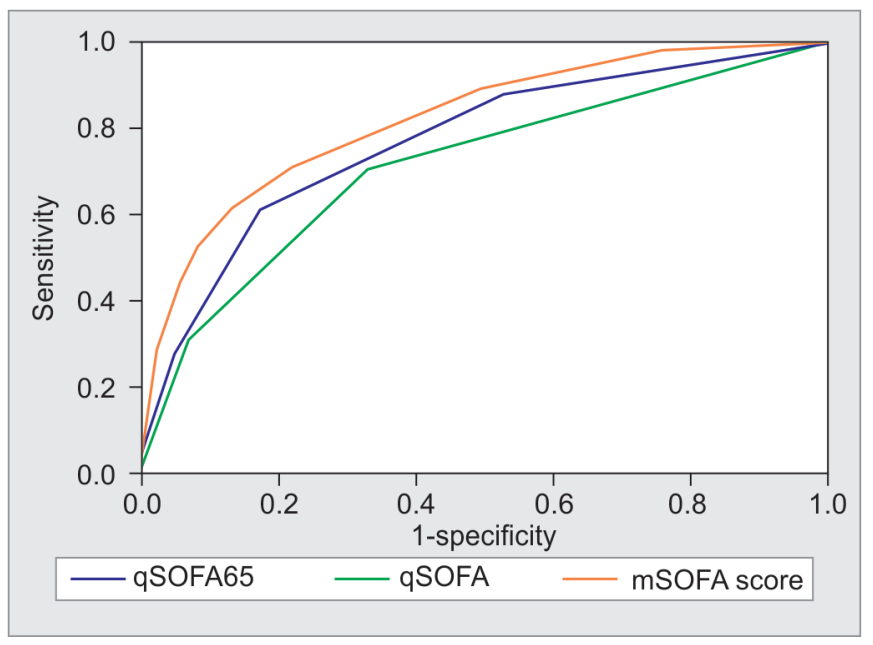

Fig. 2: mSOFA, qSOFA, and qSOFA-65 scales as detecting likelihood of ICU admission

$\geq 11$ needed further serious care and were highly likely to die or be admitted to the ICU. In support of this result, Macdonald et al. (2014) reported that patients with SOFA score $\geq 2$ needed ED admission, 


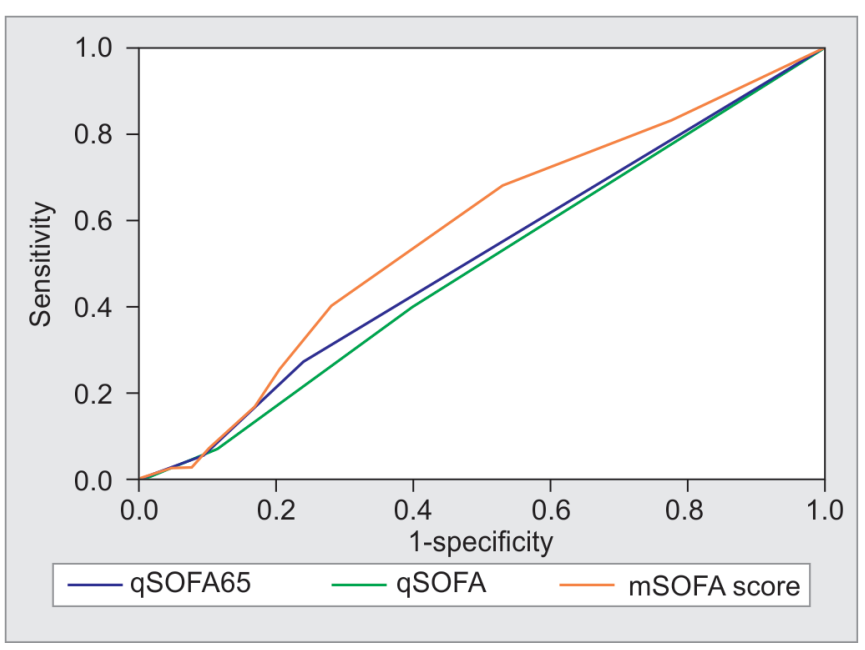

Fig. 3: mSOFA, qSOFA, and qSOFA-65 scales as detecting likelihood of general ward admission

and those with SOFA score $\geq 6$ were at risk of death. ${ }^{19}$ This result shows that the mSOFA can be used as a suitable scale for triage of nontraumatic patients in the ED. So that patients with mSOFA scores between 0 and 1 do not need ED admission, and those with scores between 2 and 6 receive green labels, and scores between 6 and 11 and higher receive red labels.

The results also showed that patients with scores of qSOFA and qSOFA- 65 between 0 and 1 needed admission to ED and further assessments. Patients with scores of 2 and higher in these two scales needed serious care and were highly likely to die or be admitted to the ICU. In line with this result, Singer et al. showed that the likelihood of death and admission to the ICU in patients with qSOFA scores $\geq 2$ were $37 \%$ and $48.2 \%$, respectively. ${ }^{15}$ Jonghoo and Jae-Uk Son showed that patients with qSOFA- 65 score $\geq 2$ were not well and were at risk of septic shock. ${ }^{17}$ This result shows that qSOFA and qSOFA-65 cannot be used as a suitable tool for identifying patients requiring admission to ED. But they can be used to triage nontraumatic patients in ED, so that patients scoring 0,1 , and $\geq 2$ in these two scales can be given green, yellow, and red labels, respectively.

The study results were showed a direct relationship between patients' exacerbating condition and higher scores in all three scales. This is confirmed in a study by Jawa et al., which showed a direct relationship between higher qSOFA scores and increased likelihood of death. ${ }^{18}$

The AUROC for mSOFA scale was found 0.924 for identifying patients at risk of death, 0.825 for identifying patients requiring admission to the ICU, and 0.569 for patients requiring transfer to other wards. This shows that this scale can be used in ED to identify critically ill patients and those at risk of death. However, this scale is not suitable for identifying patients requiring transfer to other wards. Researchers found no study investigating the accuracy of mSOFA in identifying critically ill patients in the ED. However, the SOFA has been used in some to identify critically ill patients in the ED. Macdonald reported AUROC of 0.78 for identifying patients with infectious diseases at risk of death. ${ }^{19}$ Safari reported AUROC of 0.73 for identifying patients with reduced consciousness and infection at risk of death. ${ }^{20}$ This finding shows that the mSOFA is much more suitable than SOFA for identifying the likelihood of death in nontraumatic patients presenting to ED.
For qSOFA and qSOFA-65, AUROC was 0.924 and 0.947 for identifying patients at risk of death, respectively, 0.719 and 0.773 for identifying patients requiring admission to the ICU, respectively, and 0.495 and 0.512 for identifying patients requiring transfer to other wards, respectively. These results show that compared to mSOFA, these two scales are more capable of identifying nontraumatic patients who are critically ill or at risk of death. But they are not suitable for predicting the status of patients who are not critically ill. Haydar et al. reported AUROC of 0.68 for identifying patients with infectious diseases at risk of death using qSOFA. ${ }^{21}$ Hawang et al. also reported that AUROC of 0.58 for identifying critically ill patients at admission to ED, 0.60 at 3 hours after admission, and 0.57 at 24 hours after admission. ${ }^{22}$

\section{Limitation}

The criterion for transferring patients from the ED to other wards was according to the opinion of emergency medicine and internal medicine specialists. Although the specialists' team did this very carefully, they may have made a mistake in choosing the patient ward. Therefore, this study is limited in this regard.

\section{Conclusion}

In summary, the present study showed that the likelihood of critical illness and death of nontraumatic patients referred to ED increased with increasing scores of mSOFA, qSOFA, and qSOFA-65. The cut-off point for identifying the risk of death was 6 for the mSOFA, 1 for qSOFA, and 2 for qSOFA-65. All three scales were found to be reliable for identifying nontraumatic patients at risk of death and patients requiring admission to the ICU. However, since the time and data required to complete qSOFA and qSOFA- 65 are much less than those of mSOFA, it is recommended that qSOFA and especially qSOFA-65 be used in ED to identify critically ill nontraumatic patients.

\section{ACKNOWLEDgements}

This study was part of a Master's Thesis in emergency nursing that was supported by the Nursing Care Research Center of "...". The authors thank all patients who participated in this study.

\section{References}

1. Pines JM, Griffey RT. What we have learned from a decade of ED crowding research. Acad Emerg Med 2015;22(8):985-987. DOI: 10.1111/ acem.12716.

2. Jo S, Jin YH, Lee JB, Jeong T, Yoon J, Park B. Emergency department occupancy ratio is associated with increased early mortality. J Emerg Med 2014;46(2):241-249. DOI: 10.1016/j.jemermed.2013.05.026.

3. Edmondson D, Shimbo D, Ye S, Wyer P, Davidson KW. The association of emergency department crowding during treatment for acute coronary syndrome with subsequent posttraumatic stress disorder symptoms. JAMA Intern Med 2013;173(6):472-475. DOI: 10.1001/ jamainternmed.2013.2536.

4. Hong KJ, Shin SD, Song KJ, Cha WC, Cho JS. Association between ED crowding and delay in resuscitation effort. Am J Emerg Med 2013;31(3):509-515. DOI: 10.1016/j.ajem.2012.09.029.

5. Watts H, Nasim MU, Sweis R, Sikka R, Kulstad E. Further characterization of the influence of crowding on medication errors. J Emerg Trauma Shock 2013;6(4):264-270. DOI: 10.4103/0974-2700.120370.

6. Verelst S, Wouters P, Gillet JB, Van den Berghe G. Emergency department crowding in relation to in-hospital adverse medical events: a large prospective observational cohort study. J Emerg Med 2015;49(6):949-961. DOI: 10.1016/j.jemermed.2015.05.034. 
7. Hughes JA, Brown NJ, Chiu J, Allwood B, Chu K. The relationship between time to analgesic administration and emergency department length of stay: a retrospective review. J Adv Nurs 2020;76(1):183-190. DOI: 10.1111/jan.14216.

8. Sun $B C$, Hsia RY, Weiss RE, Zingmond D, Liang LJ, Han W, et al. Effect of emergency department crowding on outcomes of admitted patients. Ann Emerg Med 2013;61(6):605-611.e606. DOI: 10.1016/j. annemergmed.2012.10.026.

9. Tekwani KL, Kerem Y, Mistry CD, Sayger BM, Kulstad EB. Emergency department crowding is associated with reduced satisfaction scores in patients discharged from the emergency department. West $J$ Emerg Med 2013;14(1):11-15. DOI: 10.5811/westjem.2011.11.11456.

10. Alishahi Tabriz A, Trogdon JG, Fried BJ. Association between adopting emergency department crowding interventions and emergency departments' core performance measures. Am J Emerg Med 2019;38(2):258-265. DOI: 10.1016/j.ajem.2019.04.048.

11. Ebrahimian AA, Khalesi N, Mohammadi G, Tourdeh M, Naghipour M. Transportation management in pre-hospital emergency whit physiological early warning scores. J Health Admin 2012;15(49):7-13.

12. Ebrahimian A, Seyedin H, Jamshidi-Orak R, Masoumi G. Exploring factors affecting emergency medical services staffs decision about transporting medical patients to medical facilities. Emerg Med Int 2014;2014:215329.

13. Grissom CK, Brown SM, Kuttler KG, Boltax JP, Jones J, Jephson AR, et al. A modified sequential organ failure assessment score for critical care triage. Disaster Med Public Health Prep 2010;4(4):277-284. DOI: 10.1001/dmp.2010.40.

14. Seymour CW, Liu VX, Iwashyna TJ, Brunkhorst FM, Rea TD, Scherag $A$, et al. Assessment of clinical criteria for sepsis: for the third international consensus definitions for sepsis and septic shock (sepsis-3). JAMA 2016;315(8):762-774. DOI: 10.1001/jama.2016.0288.

15. Singer AJ, Ng J, Thode HC, Spiegel R, Weingart S. Quick SOFA scores predict mortality in adult emergency department patients with and without suspected infection. Ann Emerg Med 2017;69(4):475-479 DOI: 10.1016/j.annemergmed.2016.10.007.

16. Garbero RF, Simões AA, Martins GA, Cruz LVF, von Zuben VGM. SOFA and qSOFA at admission to the emergency department: diagnostic sensitivity and relation with prognosis in patients with suspected infection. Turk J Emerg Med 2019;19(3):106-110. DOI: 10.1016/j. tjem.2019.05.002.

17. Lee J, Song JU. Performance of a quick SOFA- 65 score as a rapid sepsis screening tool during initial emergency department assessment: a propensity score matching study. J Crit Care 2020;55:1-8. DOI: 10.1016/j.jcrc.2019.09.019.

18. Jawa RS, Vosswinkel JA, McCormack JE, Huang EC, Thode Jr HC, Shapiro MJ, et al. Risk assessment of the blunt trauma victim: the role of the quick sequential organ failure assessment score (qSOFA). Am J Surg 2017;214(3):397-401. DOI: 10.1016/j.amjsurg.2017. 05.011 .

19. Macdonald SPJ, Arendts G, Fatovich DM, Brown SGA. Comparison of PIRO, SOFA, and MEDS scores for predicting mortality in emergency department patients with severe sepsis and septic shock. Acad Emerg Med 2014;21(11):1257-1263. DOI: 10.1111/acem.12515.

20. Safari S, Shojaee M, Rahmati F, Taghizadieh A, Baratloo A, Fattah FHR, et al. Accuracy of SOFA score in prediction of 30 day outcome of critically ill patients. Turkish J Emerg Med 2016;16(4):146-150. DOI: 10.1016/j.tjem.2016.09.005.

21. Haydar S, Spanier M, Weems P, WoodS, Strout T. Comparison of QSOFA score and SIRS criteria as screening mechanisms for emergency department sepsis. Am J Emerg Med 2017;35(11):1730-1733. DOI: 10.1016/j.ajem.2017.07.001.

22. Hwang SY, Jo IJ, Lee SU, Lee TR, Yoon H, Cha WC, et al. Low accuracy of positive qSOFA criteria for predicting 28 day mortality in critically ill septic patients during the early period after emergency department Presentation. Ann Emerg Med 2018;71(1):1-9.e2. DOI: 10.1016/j. annemergmed.2017.05.022. 